\title{
Evidence for the Luttigger liquid density of states in transport across the incompressible stripe at fractional filling factors
}

\author{
E.V. Deviatov, ${ }^{1, \text { * }}$ A.A. Kapustin, ${ }^{1}$ V.T. Dolgopolov,${ }^{1}$ A. Lorke,${ }^{2}$ D. Reuter,${ }^{3}$ and A.D. Wieck ${ }^{3}$ \\ ${ }^{1}$ Institute of Solid State Physics RAS, Chernogolovka, Moscow District 142432, Russia \\ ${ }^{2}$ Laboratorium für Festkörperphysik, Universität Duisburg-Essen, Lotharstr. 1, D-47048 Duisburg, Germany \\ ${ }^{3}$ Lehrstuhl für Angewandte Festkörperphysik, Ruhr-Universität Bochum, \\ Universitätsstrasse 150, D-44780 Bochum, Germany
}

(Dated: October 22, 2018)

\begin{abstract}
We experimentally investigate transport across the incompressible stripe at the sample edge in the fractional quantum Hall effect regime at bulk filling factors $\nu=2 / 3$ and $\nu=2 / 5$. We obtain the dependence of the equilibration length, that is a phenomenological characteristics of the transport, on the voltage imbalance and the temperature, at high voltage imbalances. These dependencies are found to be of the power-law form, which is a strong evidence for the Luttigger liquid density of states.

PACS numbers: $73.40 . \mathrm{Qv} 71.30 .+\mathrm{h}$
\end{abstract}

Edge states [1] (ES) in the integer quantum Hall effect (IQHE) regime are arising at the intersections of the Fermi level and Landau levels, bent up by the edge potential. The Landauer-Buttiker formalism [2], which is based on the concept of one-dimensional current-carrying channels, was proposed to calculate electron transport through ES. The concept of ES and Buttiker formulas can also be generalised to the transport along the edge in the fractional quantum Hall effect (FQHE) regime [3, 4]. Details of the inter-ES scattering, however, depend on the edge structure of the two-dimensional electron liquid.

In the IQHE regime the sample edge can be regarded as smooth - the edge potential variation is smaller than the cyclotron gap on the scale of the magnetic length. The smooth sample edge is a set of the stripes of compressible and incompressible electron liquid [5]. This picture was confirmed in imaging experiments [6] and in experiments on transport across the sample edge in quasi-Corbino geometry [7]. The latter ones, both as the experiment [8], demonstrate that the inter-ES scattering is mostly determined by the energetic structure at the sample edge.

It is a common belief that FQHE is determined by the electron-electron interactions [9]. Also, because of smaller spectrum gaps, a sharp edge can be realized. ES in this case are regarded as the realization of the onedimensional electron liquid, also known as chiral Luttinger liquid [10]. A characteristic feature of the Luttinger liquid is the gapless collective excitation spectrum [10, 11], that is determined by the hierarchical structure of the fractional ground state [4]. The excitation spectrum defines the tunnelling density of states, which takes a form $D(E) \sim E^{1 / g-1}$, where $g$ is of universal value $g=1 / \nu$. Experimental investigations were made in the cleaved-edge overgrowth technique [12, 13], that is the best candidate for the sharp sample edge realization. They demonstrate power-law $I-V$ curves for tunnelling into the fractional edge with exponents $g$ that behave as $1 / \nu$ for different $\nu$ from $1 / 4$ to 1 .
It is clear, that the gate-defined electrostatic edge can not be regarded as sharp, even for FQHE. Like in the integer case [5], the sample edge is a set of compressible and incompressible electron liquid [3]. It was shown in numerical calculations [14], that the structure of the compressible/incompressible stripes arises at the edge width of the order of 5-6 magnetic lengths, and, therefore, is present for most of real potentials. Co- and counter- propagating excitation branches exist at the edges of the incompressible stripes [14, 15], leading to the edge excitation spectrum resembling neutral acoustic modes [16]. Transport between two smooth fractional edges was tested in experiments [17] in quantum point contacts (QPC), where the power-low $I-V$ curves were reported and the influence of the potential profile in QPC on the $g$ values [15] was demonstrated.

There is, however, a possibility to investigate effects of the neutral edge modes directly. It is the investigation of transport across a single incompressible stripe at one sample edge. That allows to remove the influence of the exact form of the potential profile, leaving the effects of the neutral excitation modes to prevail in the tunnel density of states. Investigations could be performed in the quasi-Corbino sample geometry [7], which has the following advantages: (i) etched mesa edge allows to create the potential profile of the intermediate strength, where small widths of the stripes simplify neutral edge modes excitation; (ii) split-gate with well-defined stripe structure allows to separately contact stripes in the gate-gap; (iii) Corbino topology provides direct measurements of the electron transport across the incompressible stripes. This situation, up to our knowledge, was not in focus of both experimental and theoretical investigations before.

Here we experimentally investigate transport across the incompressible stripe at the sample edge in the fractional quantum Hall effect regime at bulk filling factors $\nu=2 / 3$ and $\nu=2 / 5$. We obtain the dependence of the equilibration length, that is a phenomenological charac- 


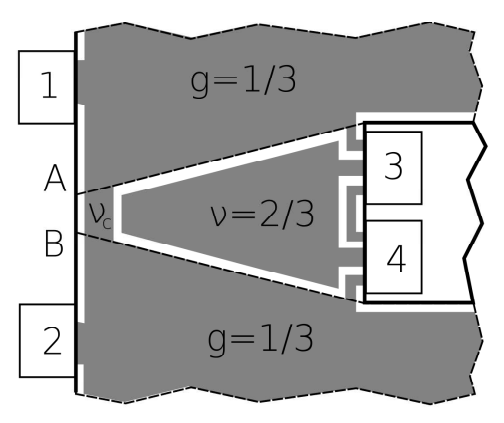

FIG. 1: Schematic diagram of the sample working area. The etched mesa edges are shown by solid lines, the dashed lines represent the split-gate edges. The gate-gap region at the outer mesa edge is denoted as AB. Light gray areas are the incompressible regions at filling factors $\nu$ (in the bulk) and $g<\nu$ (under the gate and the incompressible stripes at the mesa edges). Compressible regions (white) are at the electrochemical potentials of the corresponding ohmic contacts, denoted by bars with numbers.

teristics of the transport, on the voltage imbalance and the temperature, at high voltage imbalances. These dependencies are found to be of the power-law form, which is a strong evidence for the Luttigger liquid density of states.

Our samples are fabricated from molecular beam epitaxial-grown GaAs/AlGaAs heterostructure. It contains a 2DEG located $150 \mathrm{~nm}$ below the surface. The mobility at $4 \mathrm{~K}$ is $1.83 \cdot 10^{6} \mathrm{~cm}^{2} / \mathrm{Vs}$ and the carrier density $8.49 \cdot 10^{10} \mathrm{~cm}^{-2}$, as was obtained from usual magnetoresistance measurements. Also, magnetocapacitance measurements were performed to characterize the electron system under the gate. An interplay between two ground states [9] (spin polarised (SP) at $B=5.18 \mathrm{~T}$ and spin unpolarised (SU) at $B=4.68 \mathrm{~T}$ ) at $\nu=2 / 3$ is well developed in our samples [18] permitting the measurements at different spin configurations of the $\nu=2 / 3$ ground state.

Samples are patterned in the quasi-Corbino sample geometry [7]. Each sample has an etched region inside, providing two independent mesa edges. At bulk filling factor $\nu$ a structure of compressible and incompressible stripes exists at every edge, see Fig. 1. Some of the stripes are redirected to the other edge by depleting 2DEG under split-gate to lower filling factor $g<\nu$. The gap in the gate at the outer edge (the gate-gap region, denoted as $\mathrm{AB}$ in Fig (1) is much more narrow than at the inner one, where it is of macroscopical width $\sim 1 \mathrm{~mm}$. As a result, applying a voltage between ohmic contacts at outer and inner edges leads to the electrochemical potential imbalance across the incompressible stripe at local filling factor $\nu_{c}=g$ at the outer edge, see Fig. 11. The imbalance is a maximum $V$ at the "injection" side of the gate-gap (depends on the magnetic field direction; for example, "A" in Fig. 1). Because of the gradual equilibration across

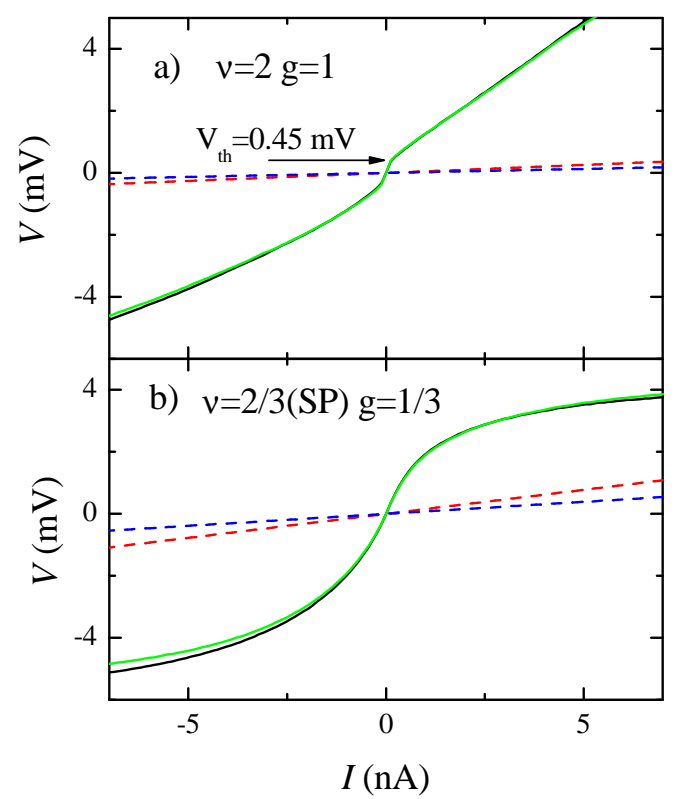

FIG. 2: (Color online) $I-V$ curves for integer filling factors $\nu=2, g=1$ (a) and fractional ones $\nu=2 / 3, g=1 / 3$ (b) for two different contact configurations. Equilibrium lines (with $\left.R_{e q}=2 ; 1 ; 6 ; 3 h / e^{2}\right)$ are shown. Magnetic field $B$ equals to $1.67 \mathrm{~T}$ for integer fillings and to $5.18 \mathrm{~T}$ for fractional ones.

the incompressible strip, the imbalance decreases while going from "A" to "B". The full current $I$, transferred across the incompressible stripe can easily be written as

$$
I=\left(V / R_{e q}\right)\left(1-\exp \left(-L_{A B} / l_{e q}\right)\right)
$$

where $l_{e q}$ is the phenomenological equilibration length, $R_{e q}$ is the equilibrium Buttiker [2, 3] resistance. Thus, $l_{e q}$ can be obtained from experimental $I-V$ curves with reasonable accuracy for $L_{A B}<<l_{e q}$ only. For the present experiment the gate-gap region is extremely narrow, $L_{A B}=0.5 \mu \mathrm{m}$, so we can expect $L_{A B}<<l_{e q}$ for any integer and fractional fillings [8].

We study $I-V$ curves in 4-point configuration, by applying $d c$ current between a pair of inner and outer contacts and measuring $d c$ voltage between another pair of inner and outer contacts. This procedure is used to remove a possible influence of the ohmic contacts on the $I-V$ curves. However, we pay a special attention to this point: several pairs of contacts at inner and outer edges of the sample allow us to study contact behaviour by 2-point magnetoresistance measurements (Corbino-contacts and non-linear ones can easily be excluded in this way). We used 4 samples with different contact recipes (resistance at low temperature is in the range $100-500 \mathrm{Ohm}$ ) and obtain similar results. The form of $I-V$ curves was additionally verified in 2-point measurements by applying dc voltage and measuring dc current. The measurements are performed in a dilution refrigerator with base temperature $30 \mathrm{mK}$, equipped with a superconducting solenoid. 
The results, presented here, are independent of the cooling cycle.

Examples of $I-V$ curves at low $(30 \mathrm{mK})$ temperature for integer $(\nu=2, g=1)$ and fractional $(\nu=$ $2 / 3(S P), g=1 / 3$ ) filling factors are shown in Fig 2, (a) and (b) correspondingly. They are strongly non-linear, but the qualitative difference is obvious: (i) for integer fillings $I-V$ is asymmetric; the positive branch of the $I-V$ trace is linear in a wide voltage range $(5 \mathrm{mV})$ and starts from the finite threshold voltage $V_{t h}=0.45 \mathrm{mV}$; (ii) for fractional fillings $I-V$ traces are practically symmetric; both branches are strongly non-linear and continuously start from the zero. This behaviour is characteristic for $I-V$ curves at all integer and fractional fillings, as investigated here as well reported before [7, 19, 20]. Also, a pronounced hysteresis is present for $\nu=2, g=1$ (not shown here), that is usual for transport between spinsplit integer ES [19], but no hysteresis can be found for fractional fillings even for different spin polarisations of the $\nu=2 / 3$ ground state.

$I-V$ curves are presented in Fig. 2 for two different contacts configurations, the difference is negligible. Thus, $I-V$ curves do reflect transport in the gate-gap across the incompressible stripe, but not the contact configuration and behaviour. Also, lines with calculated equilibrium Buttiker slopes [2, 3] $R_{e q}=h / e^{2} \nu / g(\nu-g)$ and $R_{e q}=h / e^{2} /(\nu-g)$ are shown for these contact configurations in Fig. 2. Experimental $I-V$ traces are significantly above the equilibrium lines in Fig. 2, indicating $L_{A B}<<l_{e q}$ regime in accordance with Eq. 1.

In this regime, equilibration length $l_{e q}$ can be calculated from Eq. 1, as shown in Fig. 3 for different filling factor combinations. At integer fillings $\nu=2, g=1, I-V$ curve starts from the threshold voltage $V_{t h}=0.45 \mathrm{mV}$, determined by the Zeeman splitting, because of Landau Levels bending in the incompressible $\nu_{c}=g=1$ stripe. Only voltage imbalance $V-V_{t h}$ can be redistributed [7] in Eq. 11 at high imbalances $V>V_{t h}$. We find $l_{e q}$ in this regime to be equal to $l_{e q} \sim 3 \mu \mathrm{m}$ and practically independent of $V$ in a wide $(5 \mathrm{mV})$ voltage range. This finding becomes possible only because of small $L_{A B}=0.5 \mu \mathrm{m}<<l_{e q}$, in comparison with previous reports [7] that were in the $l_{e q}<L_{A B}$ regime.

Just from the form of the $I-V$ curves for the fractional filling factors we can expect a significant dependence of the equilibration length on the voltage imbalance. This is demonstrated in Fig. 3. where $l_{e q}(V)$ are monotonically falling functions for fractional fillings. The results for $\nu=2 / 3, g=1 / 3$ are presented for two different spin orientations of the $\nu=2 / 3$ ground state [9]. Both dependencies $l_{e q}(V)$ run practically in parallel, that can be a sign, as well as the absence of the hysteresis on $I-V$ curves, that $\nu=2 / 3$ ground state polarisation is not important. The difference in value can be ascribed to the higher $\nu_{c}=1 / 3$ incompressible stripe width in the higher magnetic field at which spin-polarised

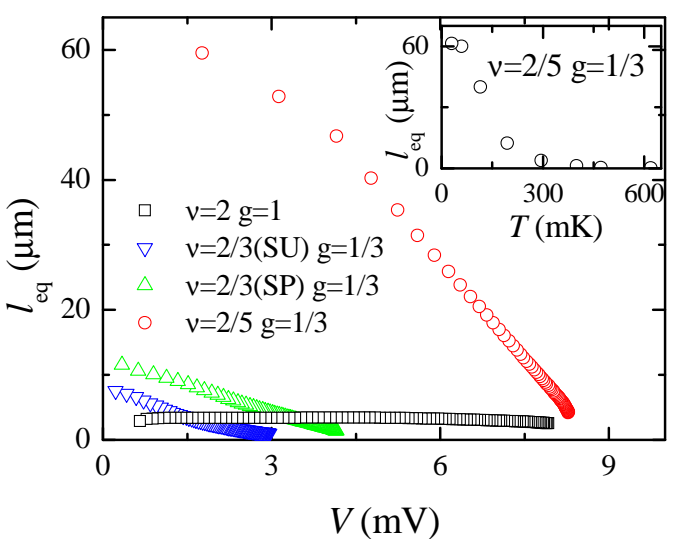

FIG. 3: (Color online) Calculated $l_{e q}$ for different filling factor combinations as function of the voltage imbalance across the incompressible stripe with corresponding $\nu_{c}=g$. Inset shows the temperature dependence of the $l_{e q}$ for $\nu=2 / 5, g=1 / 3$ $(B=7.69 \mathrm{~T})$ at voltage imbalance $V=1.6 \mathrm{mV}$.

$\nu=2 / 3$ occurs. The dependence $l_{e q}(V)$ for fractional fillings $\nu=2 / 5, g=1 / 3$ is much stronger and values of the $l_{e q}$ are much higher. Also, the temperature dependence of the $l_{e q}$ for $\nu=2 / 5, g=1 / 3$ is shown in the inset to Fig. 3 at voltage imbalance $V=1.6 \mathrm{mV}$. The form of the $l_{e q}(T)$ behaviour is independent of the $V$ value.

To analyze both $l_{e q}(V)$ and $l_{e q}(T)$ dependencies, we should mention that $l_{e q} \sim w^{-1}$, where $w$ is the transport probability. In general, it can be written as $w \sim$ $D(V, T) T_{0}(V)$ with $D$ denoting the tunnel density of states, and $T_{0}$ describing one-particle transmittance of the barrier. $T_{0}$ is determined by the potential jump in the incompressible region $\Delta$. At integer fillings $\Delta$ itself depends on the voltage imbalance $V$, because the Landau levels bending is diminishing [7] at $V>0$ in the incompressible stripe. At $V=V_{t h}$ the flat-band situation $\Delta=0$ occurs and $w$ is not sensitive to the tunnel density of states $D$, that can be seen from the constant $l_{e q}$ in a wide voltage range. At $V<0$ no flat-band situation could exist, but $D$ is the pre-exponent factor in $w$ that hardly be extracted. Thus, one-particle $T_{0}$ dominates in transport in IQHE regime.

For fractional filling factors we can not expect the flatband situation at any sign of $V$, because there are no bending of Landau levels in this case [3], which is the reason to much more symmetric $I-V$ curves. However, the significantly smaller fractional gaps [9] lead to $\Delta<<V$ in our voltage range. The transmittance $T_{0}$ can be written as $\exp \left(-C \Delta^{3 / 2} / V\right)$ in the quasiclassical triangular barrier approximation with constant $C$. In our regime $\Delta<<V$ the tunnel barrier is so thin [21], that $T_{0}(V) \sim 1$ has no influence on the transport probability $w$. Thus, $w$ is mostly determined by the tunnel density of states $D$. In Fig. 目 $w \sim l_{e q}^{-1}$ is shown as a function of the voltage imbalance $V$ (a) and the temperature (b) 

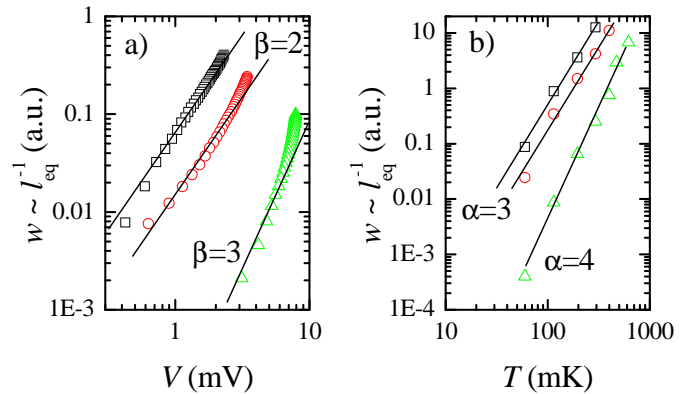

FIG. 4: (Color online) $w \sim l_{e q}^{-1}$ is shown as a function of the temperature at $V=1.6 \mathrm{mV}$ (a) and as a function of the voltage imbalance at $T=30 \mathrm{mK}$ (b) in logarithmic scales. The filling factors are $\nu=2 / 3(S U), g=1 / 3$ (squares); $\nu=$ $2 / 3(S P), g=1 / 3$ (circles); $\nu=2 / 5, g=1 / 3$ (triangles).

for different fractional filling factors in logarithmic scales. The dependencies are of a power-law behavior: $w \sim V^{\beta}$ with $\beta=2$ for $\nu=2 / 3, g=1 / 3$ (both spin polarizations) and $\beta=3$ for $\nu=2 / 5, g=1 / 3 ; w(T) \sim T^{\alpha}$ with $\alpha=3$ for $\nu=2 / 3, g=1 / 3$ and $\alpha=4$ for $\nu=2 / 5, g=1 / 3$. (The last fact, that the temperature behaviour is not activated one was also checked by plotting $w(T)$ in the Arrhenius scales.) They are the power-law dependencies $w(V) \sim V^{\beta}$ and $w(T) \sim T^{\alpha}$ with $\beta=\alpha-1$ that were predicted for tunnel density of states $D$, determined by the collective excitations [10, 11, 15]. This conclusion is also supported by the $w(V, T)$ independence of the $\nu=2 / 3$ spin polarization (see Figs. 314), because it is $T_{0}$ that is sensitive to the spin polarisation of the ground state [19] but not $D$. Thus, neutral excitation modes [16] do exist at the sample edge in the FQHE regime and determine transport across the incompressible stripe at high imbalances $V>>\Delta$.

At $\nu=2 / 3, g=1 / 3$ the neutral mode is constructed from the two excitation branches, which are counterpropagating [14] along the edges of the $\nu_{c}=1 / 3$ incompressible stripe. At $\nu=2 / 5, g=1 / 3$ we study transport across the same $\nu_{c}=1 / 3$ incompressible stripe, but the experimental exponents $\alpha, \beta$ are different. The edge of the $\nu=2 / 5$ bulk incompressible state is extremely close to $\nu_{c}=1 / 3$ stripe in this case, because of $\nu-\nu_{c}<<\nu$. Thus, we can expect some influence in $D$ also from the edge excitations of $\nu=2 / 5$ bulk incompressible state, affecting the exponents in power-low $D(V, T)$. This influence can also be responsible for the deviations from

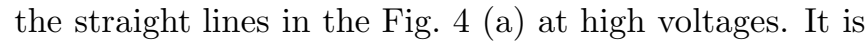
still to be calculated in further theoretical investigations.

We wish to thank D.E. Feldman for fruitful discussions and A.A. Shashkin for help during the experiment. We gratefully acknowledge financial support by the RFBR, RAS, the Programme "The State Support of Leading Scientific Schools", Deutsche Forschungsgemeinschaft, and SPP "Quantum Hall Systems", under grant LO 705/1-2. E.V.D. is also supported by MK-4232.2006.2 and Russian Science Support Foundation.

* Corresponding author. E-mail: dev@issp.ac.ru

[1] B. I. Halperin, Phys. Rev. B 25, 2185 (1982).

[2] M. Büttiker, Phys. Rev. B 38, 9375 (1988).

[3] C. W. J. Beenakker, Phys. Rev. Lett. 64, 216 (1990).

[4] A. H. MacDonald, Phys. Rev. Lett. 64, 220 (1990).

[5] D. B. Chklovskii, B. I. Shklovskii, and L. I. Glazman, Phys. Rev. B 46, 4026 (1992).

[6] E. Ahlswede, J. Weiss, K. von Klitzing, K. Eberl, Physica E 12, 105 (2002).

[7] A. Würtz, R. Wildfeuer, A. Lorke, E. V. Deviatov, and V. T. Dolgopolov, Phys. Rev. B 65, 075303 (2002); E. V. Deviatov, V. T. Dolgopolov, A. Wurtz, JETP Lett. 79, 618 (2004).

[8] G. Müller, D. Weiss, A. V. Khaetskii, K. von Klitzing, S. Koch, H. Nickel, W. Schlapp, and R. Lösch, Phys. Rev. B 45, 3932 (1992).

[9] For a review on the FQHE, see T. Chakraborty, Adv. Phys. 49, 959 (2000).

[10] Xiao-Gang Wen, Phys. Rev. B 43, 11025 (1991); Phys. Rev. Lett. 64, 2206 (1990); Phys. Rev. B 44, 5708 (1991).

[11] C.L. Kane and M.P.A. Fisher, Phys. Rev. B 46, 15233 (1992); Phys. Rev. Lett. 68, 1220 (1992).

[12] A. M. Chang, L. N. Pfeiffer, and K. W. West; Phys. Rev. Lett. 77, 2538 (1996).

[13] M. Grayson, D. C. Tsui, L. N. Pfeiffer, K. W. West, and A. M. Chang, Phys. Rev. Lett. 80, 1062 (1998); M. Hilke, D. C. Tsui, M. Grayson, L. N. Pfeiffer, and K. W. West, Phys. Rev. Lett. 87, 186806 (2001).

[14] C. d. C. Chamon and X. G. Wen, Phys. Rev. B 49, 8227 (1994).

[15] S. Conti and G. Vignale, Phys. Rev. B 54, 14309 (1996).

[16] I.L. Aleiner and L.I. Glazman, Phys. Rev. Lett. 72, 2935 (1994).

[17] S. Roddaro, V. Pellegrini, F. Beltram, G. Biasiol, and L. Sorba, Phys. Rev. Lett. 93, 046801 (2004); S. Roddaro, V. Pellegrini, F. Beltram, L. N. Pfeiffer, and K. W. West Phys. Rev. Lett. 95, 156804 (2005).

[18] V.T. Dolgopolov et. al., cond-mat/0606716

[19] E. V. Deviatov, A. Wurtz, A. Lorke, M. Yu. Melnikov, V. T. Dolgopolov, D. Reuter, A. D. Wieck, Phys. Rev. B 69, 115330 (2004).

[20] A. Wurtz, E. V. Deviatov, A. Lorke, V. T. Dolgopolov, D. Reuter, and A. D. Wieck, Physica E 22, 177 (2004).

[21] $\Delta<<V$ supports the triangular barrier approximation for any initial barrier profile, but breaks the quasiclassical limit - one-particle tunnell probability is close to one. We need only in the last fact here. 